\title{
Dissociating retention and access in working memory: An age-comparative study of mental arithmetic
}

\author{
KLAUS OBERAUER, ANKE DEMMRICH, ULRICH MAYR, and REINHOLD KLIEGL \\ University of Potsdam, Potsdam, Germany
}

\begin{abstract}
In two experiments, young and older adults solved arithmetic chain tasks with single-digit operands, with or without a concurrent memory load of three or six digits. Variables in the arithmetic tasks had to be replaced by digits from the screen or from the memory set. A task-irrelevant concurrent load impaired neither speed nor accuracy of arithmetic in younger adults. In Experiment 2, this was also true for older adults. A large decrease in arithmetic performance was observed, however, when variables in the arithmetic task had to be substituted by digits from the memory list. Older adults had specific problems with this condition in Experiment 1, where the substitution involved two successive steps, but not in Experiment 2, where the substitution from memory could be done in a single step. The results are difficult to reconcile with models assuming a common resource for storage and processing. Rather, they are compatible with the hypothesis that a concurrent memory load interferes with a processing task only during the points of access to working memory. Further, even though access to working memory was found to be the critical source of concurrent-load interference, it was found to be insensitive to the effects of adult aging.
\end{abstract}

Working memory is commonly characterized as a system for simultaneous storage and manipulation of information (Baddeley, 1986; Kyllonen \& Christal, 1990; Salthouse, 1991). Resource models of working memory assume that a limited resource is shared between the storage and the processing functions (Case, 1985; Just \& Carpenter, 1992), or that the resource is shared among the representations that must be stored and/or processed at the same time (Anderson, Reder, \& Lebiere, 1996). One corollary of this concept is that the addition of a memory load concurrent to a processing task will take away resources available for the latter, thereby reducing the speed with which the task can be performed, its accuracy, or both. This assumption is the basis for many dual-task studies of working memory. Detrimental effects of a concurrent memory load on cognitive processes are frequently found in at least one dependent variable, and when such effects are not found, this is taken as evidence for separate resources underlying the two tasks (e.g., Baddeley, 1986; Wickens, 1991).

In this paper, we want to pursue an alternative account for the tradeoff between memory ("storage") and information manipulation ("processing") in working memory. We assume that processing tasks are impaired by a concurrent working memory load if and only if the processing task requires access to contents of working memory. Many

This research was funded through the Deutsche Forschungsgemeinschaft (Grant INK 12, Project C). Correspondence should be addressed to K. Oberauer, Department of Psychology, University of Potsdam, 14415 Potsdam, Germany (e-mail: ko@ rz.uni-potsdam.de). processing tasks need information from working memory as input; this includes intermediate results from previous steps (Mayr \& Kliegl, 1993) as well as new stimulusresponse mappings given in the instruction. The processing task requires access to this information in working memory. The more elements that are held in working memory, the less efficient it should be to select the correct one as input for a process. An additional memory load will therefore reduce the discriminability of information in working memory, including the information needed for the processing task. This may slow down processing and lead to errors due to cross-talk from the irrelevant memory contents.

In contrast to resource theories, this cross-talk account of working memory capacity predicts that a memory load will not impair all concurrent processes. Instead, interference occurs specifically when the cognitive system must select a particular piece of information from working memory among other pieces. Following this account, interference between storage and processing does not arise from a shared limited capacity, but from the difficulty of selecting among the working memory contents the information that is used in a particular processing step. As a consequence, $\operatorname{cognitive~processes~that~do~not~require~retrieval~of~}$ relevant information from working memory (i.e., access to working memory) will not be impaired by the concurrent retention of task-irrelevant material in working memory.

\section{The Role of Working Memory in Mental Arithmetic}

Consider the case of mental arithmetic. The addition or subtraction of two single-digit numbers does not require 
access to working memory. No new stimulus-response mapping must be applied, and there is no need to select one element out of a set in working memory as the object of manipulation. This is different for more complex arithmetic tasks involving, for example, three-digit numbers, carries, or brackets. These tasks require the retention of intermediate results while other results are computed. Later, the intermediate results must be accessed from working memory for further manipulations or for output.

There is converging evidence that working memory capacity is a limiting factor in complex mental arithmetic tasks. Hitch (1978) showed that errors in three-digit addition tasks increase when more time and more computational steps intervene between the calculation of a resulting digit and the overt output of this digit. In a study by Logie, Gilhooly, and Wynn (1994), mental addition of two-digit numbers was impaired by articulatory suppression and by random number generation, tasks that presumably occupy the phonologicalloop and the central executive in Baddeley's (1986) model, respectively. More evidence on the role of working memory in mental arithmetic comes from a study by Anderson et al. (1996). They had participants solve equations while simultaneously remembering two, four, or six digits. Speed and accuracy of equation solving were negatively affected by increasing memory load. Memory accuracy was worse when the equation involved more symbols, relative to fewer symbols. Anderson et al. argued that the pattern of mutual interference supports the assumption that a single resource is shared between the two tasks.

All the arithmetic tasks in these studies require access to working memory at some point. In multiple-digit addition, for example, the result from the ones must be kept in memory while the tens are added. In equation solving, the results of intermediate equation transformations must be held in memory and accessed for further computations. It is possible that the effect of working memory is specific to these access steps.

The studies concerned with the contribution of working memory to simple arithmetic tasks yielded more ambiguous results. Halford, Maybery, O'Hare, and Grant (1994) used single-digit subtraction in a dual-task study with children and found a very small though significant effect of interference. They argued that the storage and the processing function of working memory draw on different resources. Klapp, Boches, Trabert, and Logan (1991, Experiment 3) found that reciting the months of the year in order interfered only minimally with single-digit addition verification. Ashcraft, Donley, Halas, and Vakali (1992) observed a considerable increase in reaction time for verification of single-digit addition tasks when participants were required either to generate words beginning with a given letter or to name four given consonants in alphabetical order simultaneously with the addition task. The interaction of the dual-task effect with problem size (i.e., the size of the addends) was not significant. Similar findings were recently reported by Lemaire, Abdi, and Fayol (1996) and by De Rammelaere, Stuyven, and Vandieren- donck (1999). Both research groups found main effects of a secondary task on verification latency in simple arithmetic tasks, but the effects did not interact with problemdifficulty variables. The absence of such interactions is consistent with the hypothesis that the load on working memory did not affect the arithmetic process itself, but a reaction time component that was constant over all difficulty levels. Access to the arbitrary mapping of "true" and "false" to the response keys is one candidate for this process (see Logan, 1979, 1980).

\section{Memory Load and Memory Access}

The mental arithmetic studies reviewed above show that performance suffers from a concurrent secondary task if the primary task requires access to working memory, whereas there is no compelling evidence that a secondary task affects cognitive processes that do not access working memory. It is not clear, however, whether the secondary task effect is in fact specific to the access function. Alternatively, the complex arithmetic tasks could simply be more difficult overall, thus requiring more of a general resource. A secondary task might consume part of the available resource, leaving enough for single-digit computation to proceed nearly unaffected, but not enough for the more complex tasks.

Relevant evidence comes from a study by Carlson, Sullivan, and Schneider (1989). They trained participants on the evaluation of logical gates. ${ }^{1}$ At test, the logical gate task was performed in combination with a memory load of three or six digits. In one condition, the memory list was irrelevant to the gate task; in another condition, values from the memory list had to be inserted for variables in the gate task. Relative to the single-task condition, the irrelevant memory load slowed gate evaluation by about $150 \mathrm{msec}$. The size of the memory load (three or six digits) affected latencies only minimally (no statistical tests were presented for these two effects). Slowing was much larger in the access conditions, and the effect of memory set size was about 10 times as large in the access condition, relative to the irrelevant memory condition. This pattern of results is difficult to reconcile with a generalresource account of dual-task effects, because the memory set should consume about equal amounts of resource in the irrelevant and in the access conditions. The interpretation is complicated, however, by the fact that the access condition required two additional cognitive operationsthe replacement of two variables in the gate task with the respective values from the memory set-which contributed an unknown amount of time to the overall latency.

Anderson et al. (1996) studied equation solving with a concurrent memory load of two, four, or six digits. In one condition the memory load was irrelevant to the equationsolving task, whereas in another condition variables in the equations had to be substituted by the first or second digit from the memory set. The size of the memory set affected problem-solving latency and accuracy in both conditions, but the effect was larger in the substitution condition. This replicates qualitatively the difference between irrelevant 
memory load and memory access from the Carlson et al. (1989) study, although quantitatively the difference was much less dramatic in the Anderson et al. experiments.

Anderson et al. (1996) explained the effect within a formal model based on ACT-R (Anderson \& Lebiere, 1998). Source activation, which functions as a limited general resource, is divided among the elements of the equation and the elements in the memory set. Activation becomes relevant at each retrieval step; this includes retrieval of arithmetic facts and retrieval of digits from the memory list. A larger memory list imposes a reduction of source activation for each element, thereby slowing and reducing the accuracy of each retrieval step. The effect is enlarged in the access condition, because this condition requires two extra retrieval steps- the retrieval of two digits to insert for the variables - in addition to the retrieval of arithmetic facts.

The study of Anderson et al. (1996) operated with quite complex arithmetic tasks that required multiple access to working memory. If their explanation for the difference between memory load and memory access holds, it should also be valid for single-digit arithmetic. The ACT$\mathrm{R}$ model, like any other general-resource model, predicts that an irrelevant memory load impairs single-digit arithmetic, and that the impairment increases with memory set size. The memory set, although irrelevant for the arithmetic task, takes away source activation, thus reducing speed and accuracy of arithmetic fact retrieval. Our alternative account, in contrast, predicts no interference of a secondary memory load with simple mental calculation as long as access to the memory set is not necessary.

\section{Working Memory in Older Age}

Older adults are known to have less working memory capacity than younger adults (see, e.g., Salthouse, 1991, 1994). Some researchers have argued that the working memory deficit is mainly a side effect of reduced mental speed in old age. This argument is based on two patterns of results: (1) Regression analyses have shown that a large part of the age-related variance in working memory capacity can be accounted for by measures of processing speed (e.g., Salthouse, 1996). (2) Old-young plots of reaction times in various tasks demonstrate that older adults are slowed relative to younger adults by a roughly constant proportional factor (e.g., Cerella, 1990).

Nonetheless, there is evidence that older adults experience problems with working memory tasks that go beyond what can be expected from general slowing alone. Mayr and Kliegl (1993) introduced the distinction between sequential and coordinative complexity to describe categories of tasks with markedly different proportional slowing factors (see also Kliegl, Mayr, \& Krampe, 1994; Mayr, Kliegl, \& Krampe, 1996). Tasks with sequential complexity can be solved by a sequence of cognitive operations, each of which requires as input only the output of the previous operation (e.g., single-digit arithmetic chain tasks). Tasks with coordinative complexity, in con- trast, require retention of and access to intermediate results (e.g., multiple-digitarithmetic or tasks with brackets).

The contrast of sequential and coordinative complexity was applied to mental arithmetic by Verhaeghen, Kliegl, and Mayr (1997). They measured time-accuracy functions of younger and older adults for arithmetic chain tasks with and without brackets. Since only single-digit operands and single-digit intermediate results were involved, the tasks without brackets could be solved by a sequence of simple arithmetic operations that required no access to working memory. The tasks with brackets, in contrast, required retention of and access to intermediate results in working memory. No age differences were observed in any of the time-accuracy function parameters for tasks with sequential complexity (i.e., no brackets). Older adults were slower, however, and reached lower asymptotic accuracy for the coordinatively complex tasks (i.e., tasks with brackets). Age equivalence in the sequential task supports the hypothesis that older adults are not slowed at all in performing simple mental arithmetic. Even the constant slowing of older adults relative to the younger found in other studies was absent here, perhaps because the time-accuracy methodology eliminated all motor components from the performance measures (for similar results see Geary \& Wiley, 1991, and Geary, Frensch, \& Wiley, 1993). The strong age effects on the coordinative tasks, on the other hand, are compatible with the idea that older adults have a specific deficit in access to working memory. Alternatively, the problems older adults experience with brackets could arise from extra demands on executive control. Arithmetic tasks with brackets cannot be solved from left to right; the problem solver must schedule his/her operations to solve the innermost brackets first. There is evidence that older adults are impaired with respect to executive functions (Mayr \& Kliegl, 2000; West, 1996). Therefore, the additional scheduling demand could cost older adults more time and lead to more errors relative to younger adults.

\section{Summary of Hypotheses}

The present study has two goals. First, we want to test the cross-talk account of interference between retention and processing in working memory against the shared resource or shared capacity account. Second, we want to illuminate the specific problems older adults had with the complex arithmetic tasks used by Verhaeghen et al. (1997). In particular, we intend to test the hypothesis that older adults have a specific deficit in their access to working memory. The two experiments presented here were designed to test the following predictions:

1. A concurrent memory load does not impair speed or accuracy of single-digit mental arithmetic as long as access to the memory set is not required for the task. This follows directly from our cross-talk account of interference between concurrent storage and processing and is in contrast to predictions from resource theories. The cross-talk account also generates the second hypothesis. 
2. Memory load has a strong effect on speed and accuracy of mental arithmetic if the task requires access to the memory set. This effect is specific to those steps in the task that involve access. Predictions 1 and 2 together claim a dissociation of retention (i.e., a concurrentworking memory load that is not accessed) and access to working memory with respect to their effects on a processing task.

3. As long as no access to working memory is required, older adults perform the arithmetic task as accurately and nearly as fast as younger adults. This hypothesis is mainly a generalization of findings from Geary and colleagues (Geary et al., 1993; Geary \& Wiley, 1991) and Verhaeghen et al. (1997).

4. Older adults are specifically impaired-more than younger adults - when access to working memory is needed to solve the arithmetic task. This was our working hypothesis regarding the source of older adult's problems with coordinative complexity, as evidenced in Verhaeghen et al. (1997).

A critical condition for the first two predictions is that the processing task does not itself require access to working memory. A critical condition for the third hypothesis is that the impact of sensorimotor processes on the latency measure is reduced as much as possible. For these reasons, we designed a task version that minimizes output requirements and involved no arbitrary mappings of internal answers to overt responses, thereby eliminating the possibility of interference between the memory load and the response mapping instructions.

\section{EXPERIMENT 1}

The arithmetic task used in this study was a chain of single-digit additions and subtractions without brackets, similar to those used by Verhaeghen et al. (1997). The task was presented in a self-paced reading mode, where participants triggered the display of the next operation by pressing the space bar. This procedure allows measuring latencies for single operations without introducing a mapping of results onto different response alternatives. For three of the six operands, variables were displayed instead of numbers, and the variable values could be read from the screen or retrieved from working memory, depending on the condition. Three conditions of working memory demand were realized. In the control condition, the arithmetic task was solved alone; variables were substituted from a display on the screen. In the memory load condition, participants had to remember a set of three digits that were irrelevant to the arithmetic task. Variables were again substituted by numbers from a screen display. In the access condition, variables were substituted by letters from a screen display, and the letters in turn served as pointers to one of the three numbers in the memory set. Thus, all three conditions required substitution of variables by numbers. Relative to the control condition, the load condition just adds a concurrent memory load, similar to classical dual-task experiments. In the access con- dition, the problem solver must retrieve information from the memory set as input for the arithmetic task. This introduces the requirement of access to working memory. Moreover, in the access condition each substitution requires two successive steps (from variables to letters on the screen, and from these letters to the numbers in memory), thereby introducing an additional executive demand. Both factors motivate our expectation that older adults will experience specific difficulties in the access condition.

\section{Method}

\section{Participants}

Eighteen younger adults $(M=21.5$ years, range $=19-25$ years $)$ and 18 older adults $(M=67.39$ years, range $=62-72$ years $)$ participated in this experiment. An equal number of males and females were assigned to each age group. One older adult dropped out after the first session and was replaced. Younger adults were students of the University of Potsdam who were recruited by bulletins posted on the campus. Most of the older adults responded to an article in a local newspaper and were participating in a psychological study for the first time. Participants were paid 36 DM (about \$20 U.S.), older adults were also reimbursed for public transportation costs.

The two age groups did not differ in total years of formal education (younger: $M=14.39, S D=1.49$; older: $M=16.03, S D=3.61$ ). On the HAWIE Digit Symbol Substitution Test, which is a measurement of perceptual speed, younger adults obtained significantly higher scores than older adults (younger: $M=61.06, S D=9.84$; older: $M=43.44, S D=7.29$ ). On the HAWIE Vocabulary test (Wechsler, 1964), the results for the two age groups did not differ (younger: $M=35.47, S D=2.45$; older: $M=34.44, S D=4.08$ ).

\section{Apparatus and Materials}

Arithmetic chain tasks with six operations (additions and subtractions) had to be solved. Problems were randomly generated with the following restrictions: Starting values, intermediate results, and final results were digits ranging from 1 to 9 . Range of operations was -1 to -7 and +1 to +7 . Participants were told about those restrictions. The request was to compute arithmetic problems as rapidly and as accurately as possible. Participants were informed that the time needed to type the answer was not critical. There were three experimental conditions and two modes of administration.

Condition 1 (control) was a control condition where problems were presented operator by operator, the first one always being a digit. Of the remaining six digits, three were replaced by variables $(X, Y$, and $Z$ ). These variables could appear randomly at each of the six operator positions; the order of variables was also randomized (Figure 1). The assignment of digits to the three variables $X, Y$, and $Z$ was displayed in a rectangle in the upper half of the screen. This rectangle was presented for the entire duration of the arithmetic problem. Participants were instructed to replace the variables with the corresponding digits. Presentation of arithmetic problems was self-paced. Pressing the space bar displayed the next operation. After the last operation, an equality sign and a question mark appeared, and participants now had to type the result for the arithmetic problem on the numeric key pad. Auditory feedback about correctness of result was given (high tone $=$ correct, low tone $=$ incorrect). If the result was incorrect, in addition to the tone the correct answer appeared in blue for $2 \mathrm{sec}$ on the screen. After finishing an item, participants could start the next item by pressing the space bar.

Condition 2 (memory load) required the retention of three digits during mental arithmetic. A memory set consisting of the letters A, $\mathrm{B}$, and $\mathrm{C}$, paired with three digits selected at random, appeared in a rectangle in the lower half of the screen for $6 \mathrm{sec}$ before the arithmetic problem appeared. After solving the arithmetic problem, par- 


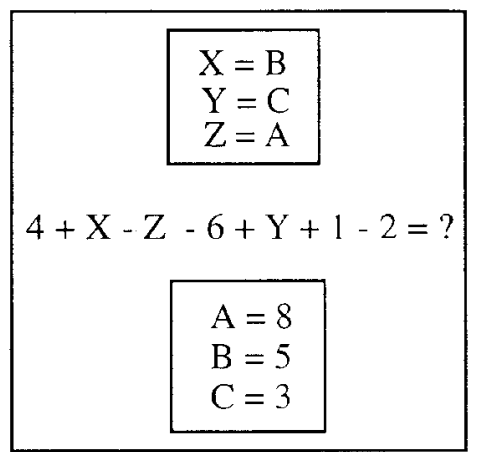

Figure 1. Experiment 1-Schematic representation of tasks in Condition 3 (access).

ticipants were probed with the three letters and asked to reproduce the corresponding digits. Apart from the added memory task, this condition was like the control condition. Participants could take their time typing the digits; auditory feedback was given for each digit. Participants were instructed to try to solve both tasks (the arithmetic problem and the memory task) correctly.

Condition 3 (access), illustrated in Figure 1, integrated the memory task with the mental arithmetic task. First, the memory set was presented for $6 \mathrm{sec}$ in a rectangle in the lower half of the screen; then the arithmetic problem started. The rectangle displayed in the upper part of the screen paired the variables $X, Y$, and $Z$ with the letters $\mathrm{A}, \mathrm{B}$, and $\mathrm{C}$ in random order. When a variable appeared in the problem, participants were instructed to pick out the corresponding letter and then retrieve the digit for that letter from their memory in order to compute the next intermediate result. Thus, the arithmetic problem could be solved only if the memory set was accessed correctly. Again, after typing the answer to the arithmetic problem, participants had to reproduce the memory set. This condition includes both a memory load and the requirement to access working memory; for brevity we refer to it as the "access condition."

There were two modes of presenting the problem. In Mode A the operators were presented one at a time. Pressing the space bar caused the last operator to disappear and the next one to be given. In problems of Mode B the already presented operators stayed on the screen until the arithmetic problem was solved. Mode B offered the possibility of recalculating the problem if it was miscalculated. After completing both the arithmetic and the memory tasks, participants indicated whether or not they had recalculated the arithmetic problem by pressing the arrow keys $(\leftarrow=$ no, $\rightarrow=$ yes $)$. We expected that older adults would show a higher frequency of recalculation, especially in the access condition. Six experimental conditions were constructed by the factorial combination of condition and presentation mode.

Data were collected using Power Macintosh 7100 and Apple Multiple Scan 17-in. monitors. Participants made their responses on the extended Macintosh keyboard (German). The experiment was programmed with the PsyScope software (Cohen, MacWhinney, Flatt, \& Provost, 1993). Stimuli were presented in green on a black background.

\section{Procedure}

The experiment was conducted in three sessions of 45-80 min each. In the first session instructions were given and trial types explained. Participants received a practice block of 10 items of each trial type. Easier conditions were practiced first to familiarize participants with the tasks. First, all conditions of Mode A were given, starting with the control condition, followed by the memory load and access condi- tions. Then, conditions of Mode B were practiced in the same order. At the end of the instruction session participants completed a short questionnaire and were assessed with psychometric tests.

Data were collected during the following two sessions. One block of every trial type consisting of 15 items had to be solved. Presentation order of blocks was counterbalanced across participants within each session. For each participant, the order of conditions was the same in the two test sessions. Conditions of the same mode were given successively. The order in which the two modes were conducted was counterbalanced across the two test sessions. At the beginning of the two test sessions, participants had the opportunity to review task descriptions and were briefly instructed again.

\section{Results}

First, accuracies for arithmetic problems and for memory sets are analyzed. Then an analysis of the probability of problems recalculated (only Mode B) is reported. Finally, we will look at the reaction times to pressing the space bar. For the condition factor, we specified two orthogonal contrasts: (1) control and memory load conditions versus the access condition, referred to as the access contrast, and (2) the control versus the memory load condition, referred to as the load contrast. A 5\% alpha level was adopted for all statistical tests.

The accuracy data were analyzed in three different ways. First, all trials were included in the analyses. Then, in the two dual-task conditions all trials with errors in the other task were excluded (i.e., arithmetic accuracies were included only for trials where the memory set was reproduced correctly, and vice versa). Finally, these accuracies were arcsin-transformed to correct for deviations from the normal distribution that are often observed for accuracies near ceiling. For all of these analyses the same pattern of significant results was obtained. Therefore, we will report only the analysis of variance (ANOVA) for accuracies of trials with correct answers to the second task.

\section{Accuracy of Arithmetic Problems}

After trials with incorrectly reproduced memory sets were excluded, probability of correctly solved arithmetic problems was subjected to a repeated measures ANOVA with mode (A vs. B) and condition (control, load, and access) as within-subjects factors and age (young vs. old) as the between-subjectsfactor. Mean accuracies are shown in the top panel of Figure 2.

There was a main effect of age, with younger adults performing better than older adults $\left[F(1,34)=12.3, M S_{\mathrm{e}}=\right.$ $0.03]$. In Mode B more problems were solved correctly than in Mode A $\left[F(1,34)=8.55, M S_{\mathrm{e}}=0.001\right]$. For both age groups, performance decreased when access to the memory set was necessary to solve the arithmetic problem, as indicated by a significant access contrast $[F(1,34)=$ $\left.78.99, M S_{\mathrm{e}}=0.01\right]$. The effect was more pronounced in older adults, as is shown by the significant interaction of the access contrast with age $\left[F(1,34)=17.10, M S_{\mathrm{e}}=\right.$ $0.01]$. There was no decrement in performance in the memory load condition relative to the control condition $(F<1$ for the load contrast). 

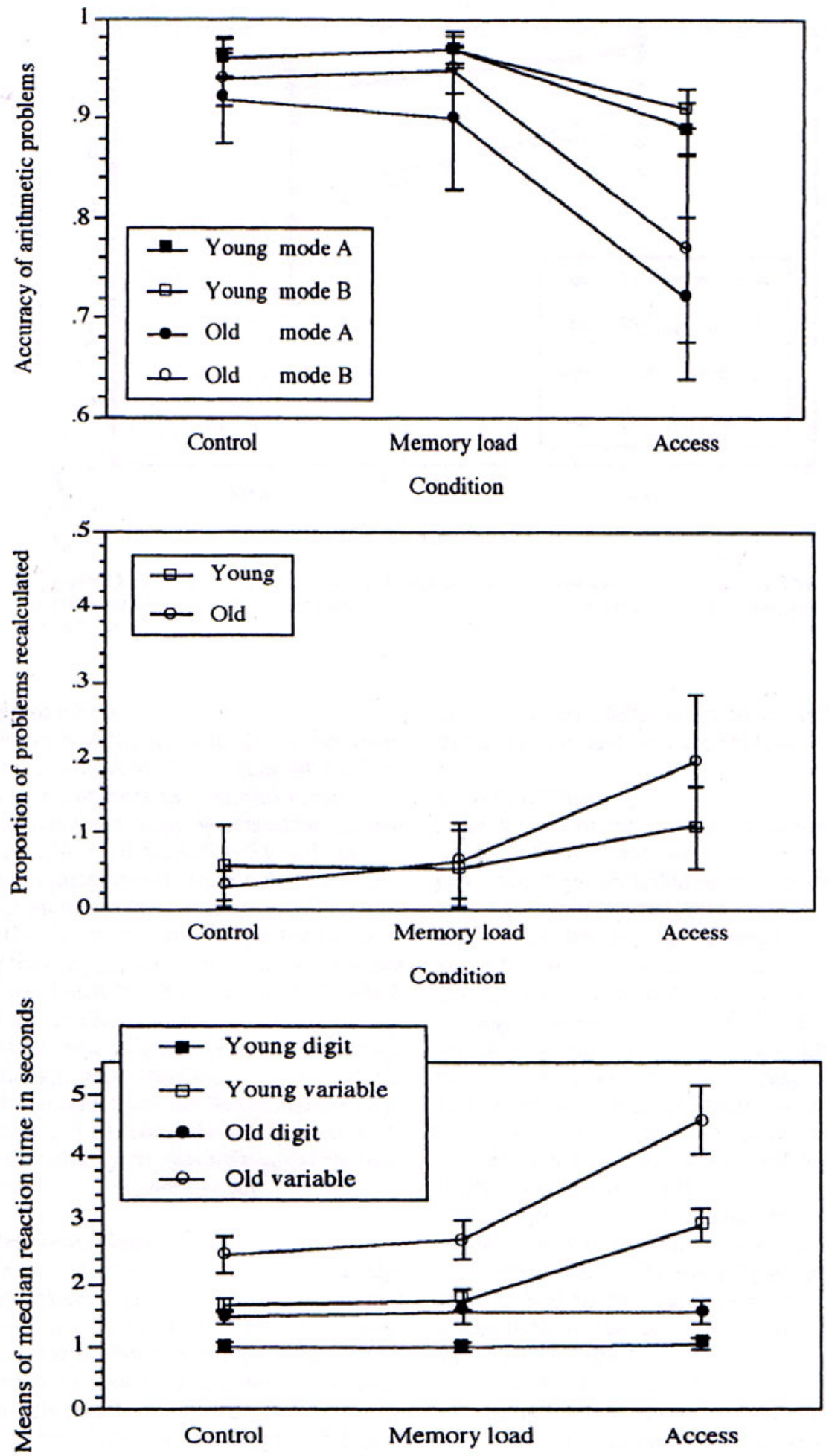

Figure 2. Experiment 1-Top panel: Accuracies of arithmetic problems for each presentation mode and age group. Middle panel: Percent of problems recalculated for each age group in presentation Mode B. Bottom panel: Means of median reaction times to variables versus digits for each age group. Error bars reflect the $95 \%$ confidence interval based on within-subjects mean squared errors. The median number of observations per participant entering into each data point ranged from 26 to 30 . 


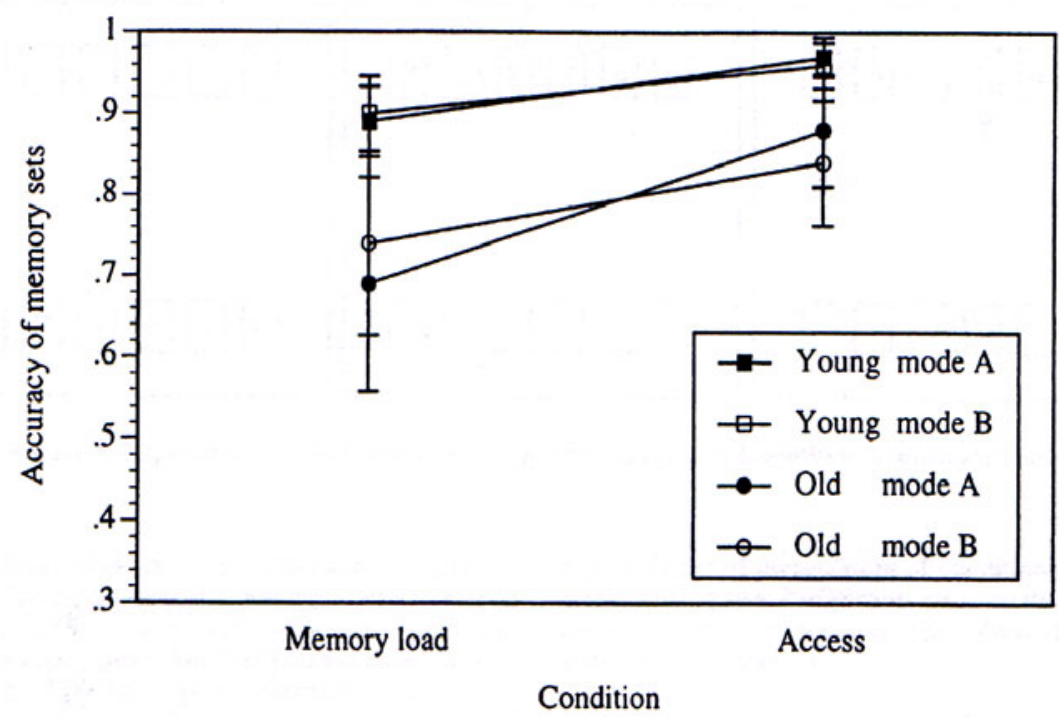

Figure 3. Experiment 1-Accuracies of memory sets for each mode and age group. The median number of observations per participant entering into each data point ranged from 26 to 28 .

\section{Accuracy of Memory Sets}

A repeated measures ANOVA with age as betweensubjects factor and mode (A vs. B) and condition (load vs. access) as within-subjects factors was conducted. Younger adults recalled more memory sets correctly than did older adults $\left[F(1,34)=10.51, M S_{\mathrm{e}}=0.07\right]$. In the access condition, more memory sets were reproduced correctly than in the memory load condition $[F(1,34)=$ 28.67, $\left.M S_{\mathrm{e}}=0.01\right]$. There was an unexpected mode $\times$ condition interaction $\left[F(1,34)=5.50, M S_{\mathrm{e}}=0.01\right.$; see Figure 3]. Recall was better for Mode B than for Mode A in the memory load condition, whereas the reverse was true for the access condition. In separate analyses by age groups, this interaction was significant only for the old adults. None of the interactions involving age was significant (all $F$ values $<3.5$ ). Thus, the previous set of results is not compromised by an age-differential tradeoff related to the accuracy of the memory set.

\section{Proportion of Problems Recalculated}

Only trials of Mode B were considered in this analysis. Percentage of problems recalculated was submitted to an age $(2) \times$ condition (3) ANOVA with repeated measures on the second factor. For the condition factor, two orthogonal contrasts were specified, as before. Results are depicted in the middle panel of Figure 2.

There was no reliable difference in the probability of problems recalculated between younger and older adults in the control and memory load conditions $(F<1)$. In these conditions both age groups recalculated fewer problems than in the more demanding access condition, as revealed by a significant access contrast $[F(1,34)=$ $\left.30.16, M S_{\mathrm{e}}=0.01\right]$. The effect of the access condition was larger for older adults, indicated by a reliable interaction of the access contrast with age $\left[F(1,34)=6.67, M S_{\mathrm{e}}=0.01\right]$.

\section{Reaction Times}

Only reaction times of trials with memory sets reproduced correctly and correct answers to the arithmetic problems were included in this analysis. For Mode B, only items without reported recalculation were considered. Individual median reaction times to pressing the space bar were computed separately for each cell of the design. Median reaction times were subjected to an age (young vs. old) $\times$ mode (A vs. B) $\times$ stimulus (digit vs. variable operators) $\times$ condition (control, load, and access) ANOVA with repeated measures on the last three factors. For the condition factor, two orthogonal contrasts were specified, as before (i.e., the access contrast defined as control and load vs. access, and the load contrast defined as control vs. load).

Averaged over all three conditions, reaction times in Mode $\mathrm{B}$ were $45 \mathrm{msec}$ faster than in Mode $\mathrm{A}[F(1,34)=$ $\left.7.30, M S_{\mathrm{e}}=0.03\right]$. The overall pattern of results was the same for both modes, and therefore we only present means across both modes for median reaction times in the bottom panel of Figure 2.

Reaction times to variables, but not to digits, increased substantially in the access condition relative to the other two conditions. The increment was larger for older adults, suggesting that the access to working memory content was selectively impaired for them. This pattern was statistically confirmed by a significant three-way interaction of the access contrast with stimulus and age $[F(1,34)=$ $\left.14.50, M S_{\mathrm{e}}=0.27\right]$. Reaction times increased in the access condition by $1.2 \mathrm{sec}$ for younger and by $2.2 \mathrm{sec}$ for older 
adults relative to the other two conditions. Reaction times to digits increased by only $51 \mathrm{msec}$ for younger and $35 \mathrm{msec}$ for older adults, a nonsignificant difference $(F=$ 2.7). Several lower order effects also reached significance. Older adults were generally slower than younger adults $\left[F(1,34)=43.66, M S_{\mathrm{e}}=1.7\right]$, and reaction times for variables were longer than those for digits $[F(1,34)=432.02$, $\left.M S_{\mathrm{e}}=0.49\right]$. The difference between reaction times to variables and to digits was larger in older age $[F(1,34)=$ $\left.20.51, M S_{\mathrm{e}}=0.49\right]$. The access contrast and its interaction with age were reliable $\left[F(1,34)=301.74, M S_{\mathrm{e}}=0.22\right.$, and $F(1,34)=16.49$, respectively $]$, as was the interaction of stimulus with the access contrast $[F(1,34)=220.33$, $\left.M S_{\mathrm{e}}=0.27\right]$.

The load contrast was also significant $[F(1,34)=$ $\left.12.88, M S_{\mathrm{e}}=0.06\right]$, as was the interaction of the load contrast with type of stimulus $\left[F(1,34)=11.46, M S_{\mathrm{e}}=0.02\right]$. Separate analyses for variables and for digits revealed the following pattern: For both age groups, the demand to remember three items during execution of the arithmetic task equally slowed down reaction times for variables $\left[F(1,34)=14.66, M S_{\mathrm{e}}=0.07\right]$. The effect was larger for older adults $(240 \mathrm{msec})$ than for younger adults $(99 \mathrm{msec})$, but the interaction with age did not reach significance $(F=2.6)$. The load contrast was marginally significant for the digits $\left[F(1,34)=4.13, M S_{\mathrm{e}}=0.02, p=.05\right]$, and it was qualified by an interaction with age $[F(1,34)=4.76$, $\left.M S_{\mathrm{e}}=0.02\right]$, indicating that older adults suffered some slowing due to the memory load $(95 \mathrm{msec})$. Younger adults, however, were completely unaffected by the memory load $(-3 \mathrm{msec})$.

To summarize the findings, it seems that reaction time for variables was increased for both younger and older adults by the demand to hold three elements in memory. When access to the working memory elements was required, older adults were affected substantially more than younger adults. In contrast, reaction time for digits was only minimally affected by a memory load or by the demand to memorize and access elements.

There was, however, a baseline difference in reaction time for younger and older adults, for digits as well as for variables. It is possible, then, to argue that the age differences observed in the most demanding access condition could be accounted for by older adults being proportionally slowed relative to younger adults (see Cerella, 1990; Kliegl et al., 1994). Proportional differences can be transformed into additive effects by logarithmic transformation of reaction times. In an ANOVA the interaction of age with the access contrast should then disappear. This was in fact the case, consistent with the proportional slowing account. The results of Experiment 2, however, will cast doubt on the hypothesis that the age $\times$ condition interaction in the present experiment is simply due to proportional slowing.

\section{Discussion}

The results of the first experiment provided partial support for the first two hypotheses outlined above. A concurrent memory load did not impair accuracy of men- tal arithmetic, and it did not slow down the performance of single-digit addition and subtraction for younger adults, as evidenced by the equivalent latencies for processing digit operations in all three conditions. There was, however, a small but reliable effect on the reaction times to digits for older adults. Memory load also slowed the processing of variables for both age groups. This could indicate that the memory list interfered with processes of visual search during the substitution process. In addition, it seems that for older adults, but not for younger adults, memory load also impaired single-digit arithmetic. Consistent with this, older but not younger adults showed somewhat lower accuracy in the arithmetic task under memory load, although this trend was not significant.

In contrast to the small or nonexistent effects of a taskirrelevant memory load, there was a large effect on both accuracy and latency when information had to be accessed from a memorized set and integrated into the arithmetic task. Access to working memory slowed down processing considerably, and it yielded a dramatic increase of errors. The increase of errors cannot be explained by failures of memory for the digits that were to be substituted, because accuracy of the arithmetic task was evaluated only for trials in which participants reproduced the complete memory set accurately at the end of the trial. Errors in the access condition could be errors of retrieval from working memory, although the memory trace itself is intact. Alternatively, errors could have occurred during substitution (e.g., forgetting of the intermediate result of the arithmetic chain or failure to select the correct number from memory) or during computation (e.g., retrieving the wrong arithmetic fact because the representation of the operand is noisy).

As predicted, the effect of the access condition was larger for older than for younger adults, on both accuracy and latency of the arithmetic task. Two factors can potentially account for this finding. First, older adults could in fact be impaired in the ability to access information in working memory. Alternatively, they could be impaired in the coordination of several successive operations. The access condition requires one more cognitive operation than the other two conditions. In the control and the memory load conditions, an operand with a variable requires two steps: Substitute the number from the screen for the variable and retrieve an arithmetic fact. The access condition requires three steps: Substitute one of the letters $\mathrm{A}, \mathrm{B}$, or $\mathrm{C}$ for the variable, substitute the corresponding digit for the letter, and retrieve an arithmetic fact. The extra operation certainly accounts for some, if not for all, of the increase in latency in both age groups. More importantly, it could also be responsible for the increase in error rate, in particular for older adults. The access condition requires that two consecutive substitutions must be internally scheduled. Scheduling of operations without guidance from the environment could place high demands on executive functions (see Hagendorf \& Sá, 1996; Mayr \& Kliegl, 1993), which might be impaired in older adults (Craik, Morris, Morris, \& Loewen, 1990; West, 1996, 
1999; but see Belleville, Rouleau, \& Caza, 1998). In this case, the specific problem of older adults in the access condition would not be due to a deficit in working memory access, but in an executive function that coordinates successive operations into a proper sequence (Mayr et al., 1996). Therefore, in Experiment 2 we implemented an access condition that required no additional substitution stage. On the basis of the thesis of an age deficit in working memory access, the large age difference should still be found in this condition.

Against our expectation, and contrary to previous findings (Geary et al., 1993; Geary \& Wiley, 1991; Verhaeghen et al., 1997), older adults were considerably slower in performing single-digit computations even with digit operators (i.e., without any form of substitution). The overall slowing factor was 1.53 . This might be interpreted as showing that retrieval of arithmetic facts from memory is not age invariant after all. Alternatively, we could account for the age difference in terms of the sensory and motor processes that still remained in the present task (e.g., pressing the space bar between operations), but that were not present in the Verhaeghen et al. study. At the moment, we cannot decide conclusively between these alternatives.

One unpredicted effect was the advantage of the access condition over the memory load condition with respect to accuracy of digit recall. A straightforward interpretation would be that participants shifted resources away from the arithmetic task to the memory task in the access condition. This is not very likely, however, because in this case we should see slowed computation with digit operations in the access condition, relative to the other conditions, which was not the case. We defer further discussion of this effect until after the second experiment.

The results of the present experiment need to be qualified in three respects. (1) The accuracy for the arithmetic tasks in the control and memory load condition was near ceiling, so it could be argued that the access $\times$ age interaction is an artifact. (2) A related concern could be that a memory load of three digits simply is not enough to produce reliable interference with the arithmetic task. Although a general resource model like ACT-R (Anderson et al., 1996) should predict a dual-task effect even with a moderate memory load, a multiple-systems model (e.g., Baddeley, 1986) could explain the absence of interference by postulating that three digits can be held entirely by a peripheral phonological slave system. (3) As noted, access to working memory was confounded with the need to coordinate two substitution operations, making the interpretation of differential age effects ambiguous. Experiment 2 was planned to remove these three problems. In addition, we intended to reevaluate the unexpected effect of memory load on arithmetic processing latency, which was observed for older but not for younger adults in the previous experiment.

\section{EXPERIMENT 2}

The second experiment replicated the basic design of Experiment 1, with two modifications. First, we increased the size of the memory set in the load and access condi- tions to six digits. This imposes a load close to the maximum span, so it is unlikely that it can be managed by a peripheral system like the phonological loop alone (Baddeley, 1996). Increasing the memory set also gives us an opportunity to compare recall accuracy for digits that were and were not accessed during problem solving. Moreover, a higher memory load should move performance away from ceiling. The second change involved the substitution procedure. In Experiment 2, the visual set of digits presented for substitution, as well as the memory set, was organized by spatial position. The digit to be selected for substitution was indicated by an arrow pointing at the relevant spatial position (see Figure 4 below). This allowed us to make demands on substitution from the screen and substitution from memory completely parallel, so that the access condition no longer required two successive substitution steps. Experiment 2 included a second access condition with a memory set of only three digits to allow a more direct comparison with Experiment 1, providing also for a contrast of different memory set sizes within the access condition.

We expected to replicate the basic results from Experiment 1 . There should be little or no effect of an additional memory load on speed and accuracy of mental arithmetic, even with a memory load of six digits. In contrast, speed and accuracy of arithmetic operations should be impaired substantially in the two access conditions. A critical question was whether the specific age-related effect of access would replicate in Experiment 2. If older adults have problems with access to working memory, we would predict that the access conditions show the largest age differences. If, on the other hand, older adults have problems with coordinating successive steps of substitution (i.e., an executive deficit), then we would expect that the specific age deficit in the access condition should not be observed in Experiment 2, where the access conditions involved only one step of substitution.

\section{Method}

\section{Participants}

In Experiment 2, participants were 16 older $(M=70.44$ years, range $=67-76$ years $)$ and 16 younger $(M=21.75$, range $=19-25$ years) adults, with equal numbers of males and females in each age group. Older adults were drawn from the Potsdam University participant pool; younger adults were students from the campus of the university. The participant fee was 36 DM (about \$20 U.S.); older adults were also reimbursed for costs of public transportation.

There were no age differences in total years of formal education (younger: $M=14.12, S D=.88$; old: $M=13.28, S D=2.88$ ). On a test of mental speed, the HAWIE Digit Symbol Substitution Test, younger adults outperformed older adults (younger: $M=62.5, S D=$ 9.13; older: $M=48.75, S D=7.78$ ). Participants also were assessed with the MWT Vocabulary Test, for which no age differences were observed (younger: $M=31.19, S D=2.69$; older: $M=32.69, S D=$ 1.89).

\section{Apparatus and Materials}

The same apparatus and type of materials were used as in Experiment 1, except that there was only one mode of presentation; each operand disappeared when a new operand was displayed.

Condition A (control) consisted of the arithmetic task alone, the problems being equivalent to those used in Experiment 1. In the 

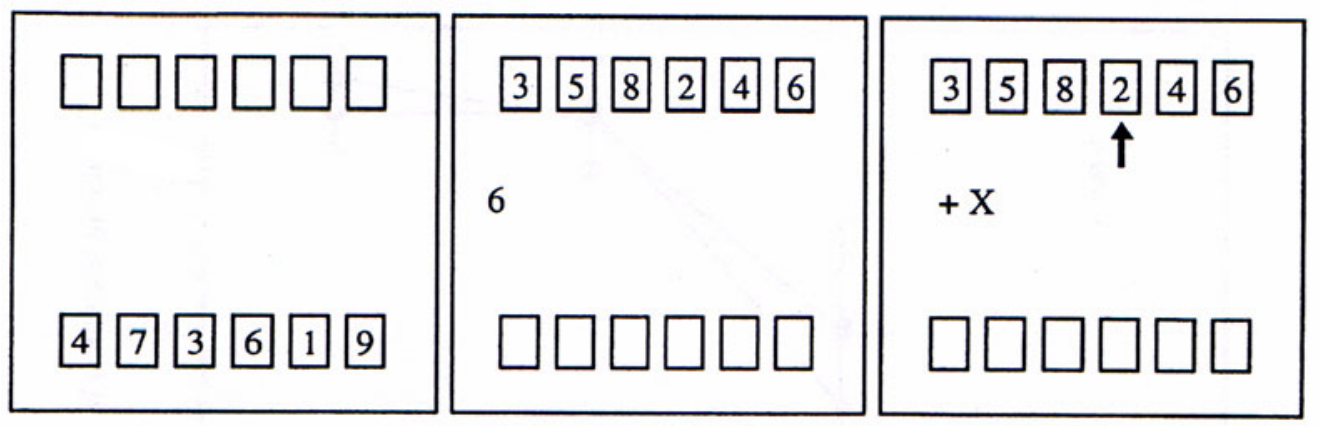

Figure 4. Experiment 2-Schematic representation of tasks in Condition B (memory load).

upper half of the screen a row of six boxes containing six digits was presented for the entire duration of the arithmetic problem. The arithmetic task was implemented as a moving windows paradigm that was self-paced by pressing the space bar (presentation Mode A from Experiment 1). Half the operators were replaced by a " $+\mathrm{X}$ " or "-X." Each position in the arithmetic chain was equally likely to be replaced by a variable. During the appearance of the $X$, a red arrow was presented on the screen that pointed to the box of the digit that was to be substituted for it.

In Condition B (memory load), a memory set of six digits was presented for $12 \mathrm{sec}$ (i.e., $2 \mathrm{sec}$ per item) in a row of boxes in the lower half of the screen prior to the appearance of the arithmetic problem (Figure 4). Then the upper row of boxes with digits appeared, and subjects performed the arithmetic task as in the control condition, again substituting three variables from the six digits on the screen. The three digits to be used for substitution were a random subset of these six digits. The digits on the screen were unrelated to the digits in the memory set. After typing the answer to the problem, participants were requested to reproduce the memory set in correct order (one digit after the other, without the opportunity to correct mistakes). For each item of the memory set, auditory feedback was given.

Condition $C$ (access $=6$ ) started with the presentation of a memory set of six digits in the box row in the upper half of the screen for $12 \mathrm{sec}$. Then, the digits disappeared, but the boxes remained on the screen. Participants now were required to retrieve three of the digits during the execution of the task and to substitute them for X. When an $\mathrm{X}$ appeared in the equation, a red arrow pointed to one of the empty boxes where the memory set had appeared. The corresponding value had to be retrieved from memory and substituted for the variable in the equation. Again, after finishing the arithmetic task, participants were asked to reproduce the memory items in correct order.

Condition D (access $=3$ ) was identical to Condition C except that the memory set consisted of only three digits (presented at the first three spatial positions of the six-box array used in the other conditions). It was presented for only $6 \mathrm{sec}$ to equate the presentation time per items over all conditions. All of the digits in the memory set were used in the arithmetic problem.

\section{Procedure}

The experiment was conducted in three sessions. In the first session participants were instructed and then practiced 10 items of each condition to become familiar with the tasks. Then, the questionnaire was filled out, and the psychometric tests were administered.

Data were collected during the following two sessions. In each of the two test sessions, one block of 15 items per condition had to be solved. Order of presentation of conditions was counterbalanced across participants. Participants received the same order of presentation in the two test sessions. They always had the opportunity to review task descriptions.

\section{Results}

As in Experiment 1, accuracies of arithmetic problems and of the memory task are reported first. Then we turn to the results for reaction times.

\section{Accuracy of Arithmetic Problems}

Only trials with memory sets reproduced completely correct were included in this analysis. Percentage of correctly solved arithmetic problems was subjected to a repeated measures ANOVA with condition (control, load, access- 6 , and access- 3 ) as within-subjects factor and age as the between-subjects factor. Three orthogonal contrasts were specified: (1) The first contrast (the access contrast) tests the two access conditions (three and six elements) against the combination of control and load conditions. (2) The second contrast (the load contrast) compares the load condition and the control condition. (3) The access (six elements) condition was tested against the access (three elements) condition by the third contrast (the setsize contrast). In the top panel of Figure 5 mean accuracies for the two age groups are depicted.

As in Experiment 1, the control condition and the memory load condition did not differ in accuracy $(F=.48)$. There was a significant drop in accuracy in the two access conditions relative to the other two conditions $[F(1,30)=$ $\left.47.91, M S_{\mathrm{e}}=0.01\right]$. Within the access conditions, performance was better with a memory set of three digits than with six digits $\left[F(1,30)=16.27, M S_{\mathrm{e}}=0.01\right]$. Nonetheless, performance was worse even when the access condition with three digits was tested alone against the control and the load conditions $\left[F(1,30)=17.28, M S_{\mathrm{e}}=0.004\right]$. There were no reliable age differences $(F=1.71)$ and no interactions involving age.

A second analysis was performed with all trials included, regardless of performance in the memory task. The results were the same as reported above, except that 

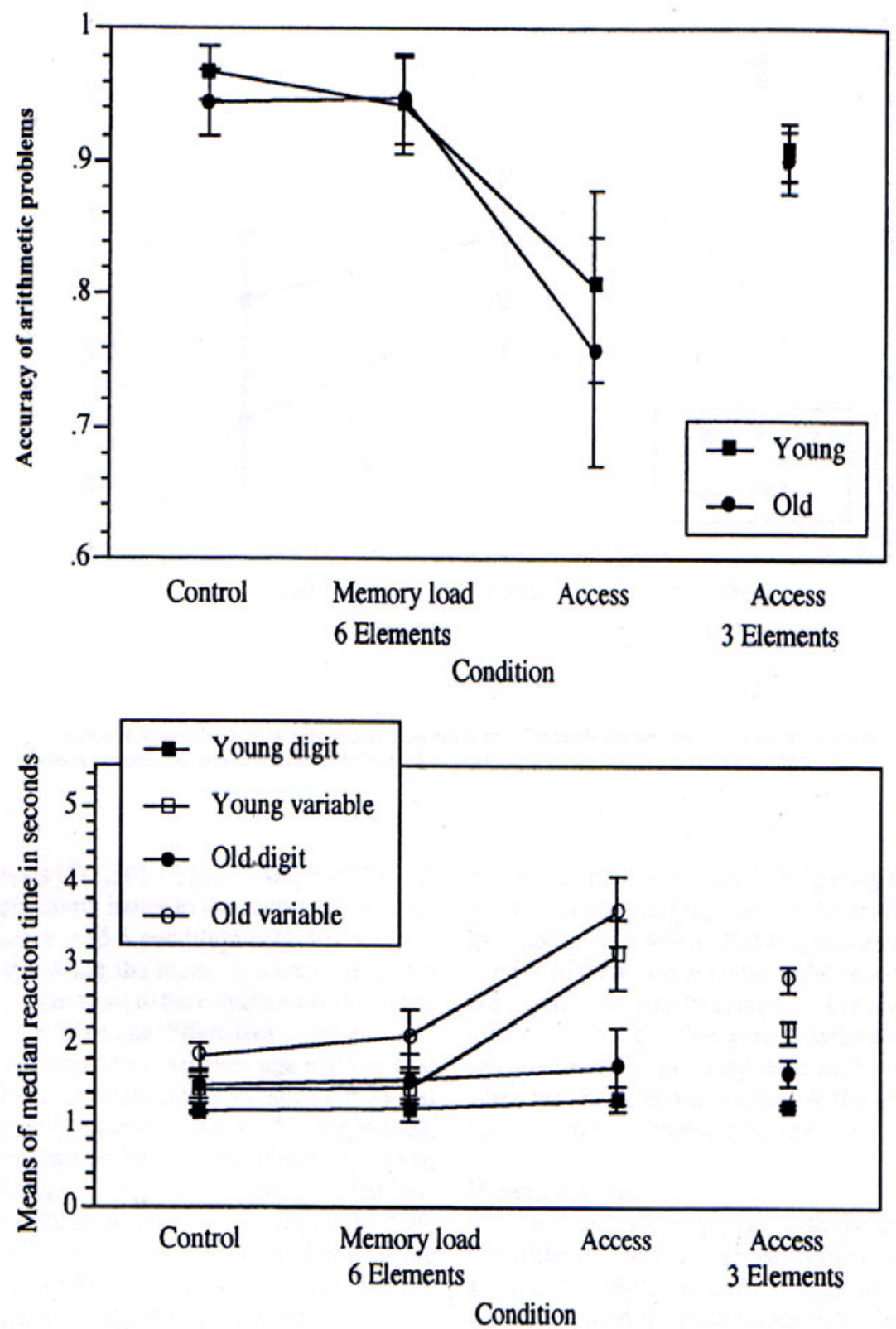

Figure 5. Experiment 2-Top panel: Accuracies of arithmetic problems for each age group. Bottom panel: Means of median reaction times to digits versus variables for each age group. Error bars reflect the $95 \%$ confidence interval based on within-subjects mean squared errors. The median number of observations per participant entering into each data point ranged from 19 to 30.

in addition there was a main effect of age $[F(1,30)=5.86$, $\left.M S_{\mathrm{e}}=0.01\right]$.

\section{Accuracy of Memory Sets}

First, only trials with the correct answer to the arithmetic task were included in the analysis. Percentage of memory sets reproduced completely correct was submitted to a repeated measures ANOVA with age as betweensubjects factor and condition (load, access-6, and access3 ) as within-subjects factor. A different set of orthogonal contrasts was specified for the condition factor in this analysis: The first compares the memory load (six digits) and access (six digits) conditions with the access (three digits) condition, and the second contrast compares the memory load (six elements) with the access (six elements) condition. Figure 6 shows mean accuracies.

Younger adults reproduced more memory sets correctly than did older adults $\left[F(1,30)=4.25, M S_{\mathrm{e}}=0.06\right]$. As expected, accuracy in the access condition with three memory items was higher than in the memory load and 


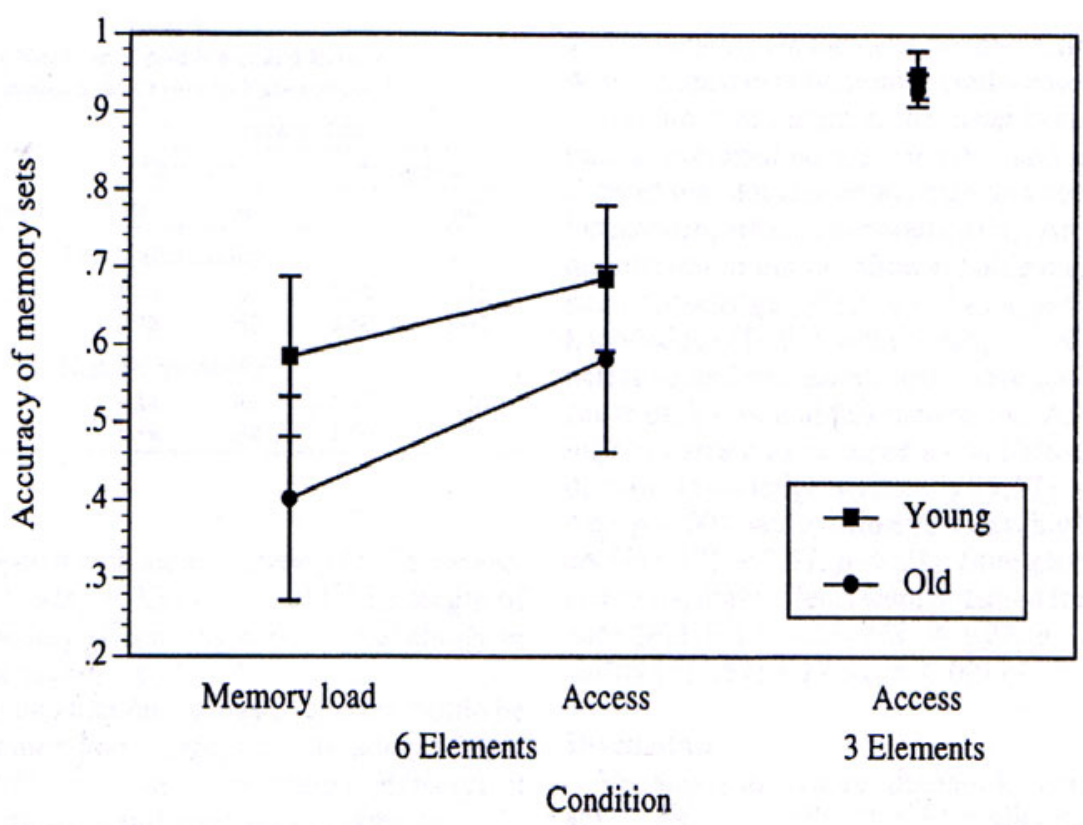

Figure 6. Experiment 2-Accuracies of memory sets for each age group. The median number of observations per participant contributing to each data point ranged from 15 to 28 .

access -6 conditions $\left[F(1,30)=131.39, M S_{\mathrm{e}}=0.02\right]$. Accuracy was significantly better in the access- 6 condition than in the memory load- 6 condition $[F(1,30)=27.85$, $\left.M S_{\mathrm{e}}=0.01\right]$, replicating the memory advantage of the access condition. In contrast to the conditions with six digits, there were no reliable age differences in the access-3 condition, but the interaction between age and the first contrast (conditions with six digits vs. access $=3$ ) just failed to reach significance $\left[F(1,30)=3.57, M S_{\mathrm{e}}=0.02\right.$, $p=.07]$. This interaction became significant, however, when the percentage of individual digits recalled correctly was taken as the dependent variable $[F(1,30)=4.99$, $\left.M S_{\mathrm{e}}=0.01\right]$. Otherwise, the results were qualitatively the same for both dependent variables and also when the analysis was repeated with all trials included.

For the next analysis, we focused on the access- 6 condition and computed the percentage of digits recalled correctly that had to be accessed versus not accessed during the arithmetic task (again, only for trials with the correct answer to the arithmetic problem). These data were subjected to an age (2) $\times$ access (2) ANOVA. Digits that had to be accessed were more likely to be recalled than digits that were not $\left[F(1,30)=10.59, M S_{\mathrm{e}}=0.001\right]$. Accessed digits were recalled with $91 \%$ accuracy by younger adults and $86 \%$ by older adults, and those not accessed were recalled with $89 \%$ (younger) and $81 \%$ (older) accuracy. When we compared those digits in the access6 condition that were not accessed with the six digits in the memory load condition, the three digits in the access- 6 condition were still reproduced better $[F(1,30)=23.35$, $\left.M S_{\mathrm{e}}=0.001\right]$. The difference, however, was reduced to 3 percentage points and was no longer significant when trials with erroneous responses to the arithmetic task were included in the analysis. This suggests that the memory advantage of the access condition relative to the memory load condition arose from two sources: (1) a memory boost specific to those digits that were actually accessed and (2) a selective sampling of easy tasks in the access condition when we restricted the analysis to the trials with correct results in the arithmetic problem.

\section{Reaction Times}

In this analysis only trials with the correct answer to the arithmetic problem as well as with memory sets reproduced completely correct were included. Medians were computed for reaction times to digits and to variables for each participant and condition. These reaction times were submitted to an age (young vs. old) $\times$ stimulus (digits vs. variables) $\times$ condition (control, load, access-3, and access-6) ANOVA. The same contrasts as for accuracies of arithmetic problems were specified. In the bottom panel of Figure 5, reaction times for digits and for variables are displayed separately for younger and older adults.

Younger adults were faster in pressing the space bar than older adults $\left[F(1,30)=9.86, M S_{\mathrm{e}}=1.43\right]$. There was a significant main effect of stimulus $[F(1,30)=524.06$, $M S_{\mathrm{e}}=0.10$ ], indicating that reaction times for variables were longer than for digits. This effect interacted with age $\left[F(1,30)=5.63, M S_{\mathrm{e}}=0.10\right]$. The difference between the control and memory load conditions, on the one hand, and the two access conditions, on the other, was also re- 
liable $\left[F(1,30)=219.52, M S_{\mathrm{e}}=0.14\right]$, as was the interaction of this contrast with stimulus $[F(1,30)=272.59$, $\left.M S_{\mathrm{e}}=0.07\right]$. Relative to the control and storage conditions there was a significant increase in reaction times for variables in the two access conditions. A separate analysis of the reaction times to digits alone revealed that reactions were also slowed in the two access conditions relative to the control and the memory load conditions $[F(1,30)=$ $\left.15.32, M S_{\mathrm{e}}=0.04\right]$, although this effect was much smaller relative to the effect on variables. The contrast differences for variables were $1.21 \mathrm{sec}$ for younger and $1.29 \mathrm{sec}$ for older adults, and those for digits were $0.1 \mathrm{sec}$ for younger and $0.25 \mathrm{sec}$ for older adults. Contrary to the results from Experiment 1, none of the effects of access on reaction times interacted with age (all $F_{\mathrm{S}}<1.5$ ).

The contrast between control condition and memory load condition interacted with stimulus $[F(1,30)=4.35$, $M S e=0.01]$, and the three-way interaction of this contrast with stimulus and age was also significant $[F(1,30)=$ $\left.5.53, M S_{\mathrm{e}}=0.01\right]$. Relative to the control condition, latencies in the memory load condition increased only for variables, and this increase ( $244 \mathrm{msec}$ ) was observed only for older adults; it was only $26 \mathrm{msec}$ for younger adults and not significant in a separate ANOVA $(F<1)$.

There was a significant difference in reaction times between the two access conditions $\left[F(1,30)=41.95, M S_{\mathrm{e}}=\right.$ $0.18]$. This effect interacted with stimulus $[F(1,30)=$ $\left.120.98, M S_{\mathrm{e}}=0.04\right]$. In the access conditions, reaction times for variables were longer when six elements had to be maintained in working memory than when only three elements had to be maintained. There was no reliable difference between the two access conditions in reaction times to the digits.

A final analysis of reaction times in the two access conditions revealed serial position effects in the access- 6 conditions. Items early in the list were accessed faster, as indicated by a linear trend $\left[F(1,30)=4.89, M S_{\mathrm{e}}=1.76\right]$, and items at both ends of the list were accessed faster than those in the middle, as shown by a strong quadratic trend $\left[F(1,30)=22.75, M S_{\mathrm{e}}=0.48\right]$. While the linear trend speaks in favor of a serial search of the memory set, the advantage of the last items in the list can be better explained by direct access from the spatial cue to the corresponding memory item. The advantage of items at both ends of the list can be explained by the higher discriminability of the end items in spatial arrays (Healy, 1977).

\section{Discussion}

Several important findings from Experiment 1 could be replicated and extended in the present experiment. First, there was no effect of an additional memory load on either accuracy of mental arithmetic or on latency for executing single steps when the operators were digits. The slight increase in latency for the variables that was already observed in Experiment 1 was now restricted to the older adults. The small effect of memory load on latency for digits observed for older adults in Experiment 1 was not replicated here. This is remarkable because a memory load of six digits is close to the participants' digit span, in particular that of the older adults. From the perspective of a general resource theory, this implies that the memory task should have consumed most of the available resources. Note that the reaction time analysis included only trials where the memory set was in fact reproduced perfectly; this rules out the hypothesis that participants could have sacrificed the memory task for maintaining a high level of performance in the arithmetic task.

Second, accuracy of mental arithmetic dropped substantially when access to the memory set was required. At the same time, latencies for the steps that actually involved access increased. As a new finding, these two parallel effects both interacted with memory set size. This interaction was not present in the latencies for processing digit operators. The pattern of results strongly indicates that processing is specifically impaired at the moment where an element must be retrieved from working memory, and that this impairment is related to the number of elements in working memory. Since the set size did not affect processing of the digits, it is difficult to argue that the access condition as a whole is different from the other two conditions in some respect (e.g., requiring more or different resources overall).

Third, there was again an advantage for the access condition (with six digits) over the memory load condition with respect to recall. This advantage seems to be specific to the digits that were actually retrieved during problem solving. In our view, the most plausible interpretation of the advantage is that each successful retrieval adds strength to the retrieved digit (see, e.g., Anderson \& Lebiere, 1998). Since the access advantage did not affect the digits that were not accessed in the unselected sample of trials, there is no compelling evidence for a resource tradeoff in favor of the memory task in the access condition.

In contrast to the first experiment, however, older adults did not appear to be specifically impaired in the access condition. Their accuracy level was comparable to that of the younger participants, and the degree of slowing they experienced on the variables in the access condition was in fact less than can be expected from a general proportional slowing factor. ${ }^{2}$ This result is clearly incompatible with the third hypothesis formulated in the introduction. Older adults do not seem to have a specific deficit in access to working memory.

\section{GENERAL DISCUSSION}

\section{Implications for Models of Working Memory}

The results of both experiments were in general agreement with the hypothesis that interference between a processing task and a concurrent memory load arises specifically when working memory is accessed. There is very little interference between a processing task and working memory contents that are concurrently retained but need not be accessed. 
This pattern of dual-task effects is difficult to reconcile with single-resource models of working memory. Resource models generally assume that short-term memorization of information concurrently with manipulation of information poses a load on resources, reducing the speed and efficiency in arithmetic processing. This prediction is made explicit in one particularly elaborated generalresource model, the ACT-R model presented by Anderson et al. (1996). Our data provide evidence against this prediction. One could argue that, for some reason, the memorization of three or six digits requires only very few resources. However, the resource account would then be unable to explain the strong effect of concurrent memory load in the access conditions. The overall amount of both "storage" and "processing" were identical in the memory load and the access conditions (at least in Experiment 2, where no double substitution was necessary), so that the specific problems observed in the access conditions can be explained neither by the addition of "storage" and "processing" demands on a common resource nor by their mutual competition for the same resource. This argument also holds for models with separate resource pools for large content domains (e.g., Shah \& Miyake, 1996), because the memory sets and the arithmetic tasks came from the same domain.

A model of working memory proposing multiple components (Baddeley, 1986; Schneider \& Detweiler, 1987) can cope with the present data somewhat better than a single-resource account, simply because it allows for more free parameters. With the model of Baddeley (1986), for example, one could argue that the three or six digits in the memory load condition are held exclusively by the phonological loop, while the central executive deals with the arithmetic task. In our reading of the theory, this would stretch the capacity of the phonological loop considerably. According to Baddeley and Hitch (1974), the phonological loop can hold a moderate number of digits without interfering with the work of the central executive. With a load close to the person's span, they argued, the central executive must support the slave system, which leads to interference with the primary task. In our Experiment 2, however, a load of six digits still did not impair mental arithmetic in any measurable way. Perhaps more important is the contrast with the access condition. Baddeley and Hitch proposed that the central executive is needed for retrieval from the rehearsal loop, for example, to interpret degraded phonological traces. This idea, if elaborated, would bring the Baddeley model very close to the approach we favor, because it attributes capacity limitations to the mechanism of access to working memory contents.

We interpret the present findings as support for a conception that attributes capacity limitations of working memory to cross-talk between activated representations. Cross-talk occurs when one of a set of available elements in working memory must be selected to serve as input for a cognitive operation (e.g., the role of the second addend in an addition). At this point, the retrieval cues can match not only the target element but also other elements in working memory, resulting in slowed and error-prone retrieval. On the other hand, as long as no selection from memory is needed, there will be no interference from a concurrent memory load. In arithmetic chain tasks without carries or brackets, this is the case: Each step in the computation needs as input only the operator, which is given perceptually, and the result of the previous operation, which is still in the focus of attention (Garavan, 1998; McElree, 1998). Selection of information from memory is required only in the access conditions. This is where we observed pronounced effects of memory load. To conclude, the impact of a concurrent short-term memory task on cognitive processing seems to be due not to an overload of shared resources, but to cross-talk between competing memory elements when the processing task requires access to the contents of working memory.

\section{Implications for Cognitive Aging}

With respect to the effect of aging on working memory, the age $\times$ condition interactions on both reaction time and accuracy data in Experiment 1 are compatible with the hypothesis that older adults are specifically impaired in their ability to access information in working memory. This interpretation, however, is questioned by the results of Experiment 2, where there was no evidence that older adults had a particular performance deficit in the access conditions relative to the memory load and the control conditions. The comparison of the age $\times$ condition interactions on reaction times over both experiments also calls into question an account based on proportional slowing. Although the data in Experiment 1 were consistent with a single proportional factor relating reaction times of older and younger participants, this was not the case in Experiment 2 . The age effect on reaction times was better characterized as an additive than a proportional constant in the second experiment. If we take the data pattern in Experiment 2 as a baseline, the age $\times$ condition interaction observed in Experiment 1 must be interpreted as reflecting a specific difficulty for older adults in that experiment's access condition.

One feature that distinguished the two experiments was the double substitution step in Experiment 1, which was not required in Experiment 2. The coordination of two substitution steps could have demanded executive control to perform the two steps smoothly in the correct order. Similar demands on executive control could be responsible for the specific problems older adults experienced when arithmetic tasks included embedded brackets (Verhaeghen et al., 1997). A task with two embedded pairs of brackets-for example, $2+((5-3)+1) \ldots$ requires scheduling of three successive steps: Solve the inner pair of brackets $(5-3)$, solve the outer pair of brackets $(2+1)$, and resume the computation in sequence $(2+3)$. This is an executive demand analogous to that involved in the double substitution of Experiment 1. Interestingly, a number of other results also strongly suggest disproportional age differences when coordination of 
interrelated processing steps becomes necessary (Kliegl et al., 1994; Mayr \& Kliegl, 1993; Mayr et al., 1996). Thus, rather than a deficit in access to working memory, the specific problem of older adults in Experiment 1 probably reflects an executive-control deficit.

A second aspect of performance that yielded substantial age differences in both experiments was the accuracy of recalling the digit lists. This points to a deficit in the retention function of working memory in older age, consistent with recent findings by Belleville et al. (1998). We cannot rule out, however, that the memory impairment of older adults was due to the interfering arithmetic task. Several studies (summarized in Craik, Anderson, Kerr, \& $\mathrm{Li}, 1995)$ found scant age-related decrements in immediate forward serial order recall of digits in the absence of distraction.

We conclude that there are two likely sources of age differences in working memory. One is the ability to keep information in memory, in particular during a distracting task. The other is an executive control function, in particular the ability to coordinate successive cognitive operations. This proposal is speculative, but in line with previous research (Mayr \& Kliegl, 1993; Mayr et al., 1996; Verhaeghen et al., 1997). Finally, and maybe most important from an aging perspective, is the conclusion that access to the contents of working memory, although highly demanding even for young adults, is not the locus of older adults' impairment in working memory.

\section{REFERENCES}

Anderson, J. R, \& Lebiere, C. (1998). The atomic components of thought. Mahwah, NJ: Erlbaum.

Anderson, J. R., Reder, L., \& Lebiere, C. (1996). Working memory: Activation limits on retrieval. Cognitive Psychology, 30, 221-256.

Ashcraft, M. H., Donley, R. D., Halas, M. A., \& VAKali, M. (1992). Working memory, automaticity, and problem difficulty. In J. I. D. Campbell (Ed.), The nature and origins of mathematical skill (pp. 301329). Amsterdam: Elsevier.

BADDELEY, A. D. (1986). Working memory. Oxford: Oxford University Press, Clarendon Press.

Baddeley, A. D. (1996). Exploring the central executive. Quarterly Journal of Experimental Psychology, 49A, 5-28.

Baddeley, A. D., \& Hitch, G. J. (1974). Working memory. In G. H. Bower (Ed.), The psychology of learning and motivation (Vol. 8, pp. 47-90). New York: Academic Press.

Belleville, S., Rouleau, N., \& Caza, N. (1998). Effect of normal aging on the manipulation of information in working memory. Memory \& Cognition, 26, 572-583.

Carlson, R. A., Sullivan, M. A., \& Schneider, W. (1989). Practice and working memory effects in building procedural skill. Journal of Experimental Psychology: Learning, Memory, \& Cognition, 15, $517-$ 526.

CASE, R. (1985). Intellectual development: Birth to adulthood. Orlando, FL: Academic Press.

Cerella, J. (1990). Aging and information processing rate. In J. E. Birren \& K. W. Schaie (Eds.), Handbook of the psychology of aging (3rd ed., pp. 201-221). San Diego: Academic Press.

Cohen, J. D., MacWhinney, B., Flatt, M., \& Provost, J. (1993). PsyScope: An interactive graphic system for designing and controlling experiments in the psychology laboratory using Macintosh computers. Behavioral Research Methods, Instruments, \& Computers, 25, 257 271.
Craik, F. I. M., Anderson, N. D., Kerr, S. A., \& Li, K. Z. H. (1995). Memory changes in normal ageing. In A. D. Baddeley, B. A. Wilson, \& F. N. Watts (Eds.), Handbook of memory disorders (pp. 211-241). Chichester, U.K.: Wiley.

Craik, F. I. M., Morris, L. W., Morris, R. G., \& Loewen, E. R. (1990). Relations between source amnesia and frontal lobe functioning in older adults. Psychology \& Aging, 5, 148-151.

De Rammelaere, S., Stuyven, E., \& Vandierendonck, A. (1999). The contribution of working memory resources in the verification of simple mental arithmetic sums. Psychological Research, 62, 72-77.

GaraVan, H. (1998). Serial attention within working memory. Memory \& Cognition, 26, 263-276.

Geary, D. C., Frensch, P. A., \& Wiley, J. G. (1993). Simple and complex mental subtraction: Strategy choice and speed-of-processing differences in younger and older adults. Psychology \& Aging, 8, 242256.

Geary, D. C., \& Wiley, J. G. (1991). Cognitive addition: Strategy choice and speed-of-processing differences in young and elderly adults. Psychology \& Aging, 6, 474-483.

Hagendorf, H., \& SÁ, B. (1996). Coordination in visual working memory. Psychological Research, 58, 294-306.

Halford, G. S., May bery, M. T., O'Hare, A. W., \& Grant, P. (1994). The development of memory and processing capacity. Child Development, $\mathbf{6 5}, 1338-1356$.

HeALY, A. (1977). Pattern coding of spatial order information in shortterm memory. Journal of Verbal Learning \& Verbal Behavior, 16, 419-437.

HiтcH, G. J. (1978). The role of short-term working memory in mental arithmetic. Cognitive Psychology, 10, 302-323.

Just, M. A., \& CARPENTER, P. A. (1992). A capacity theory of comprehension: Individual differences in working memory. Psychological Review, 99, 122-149.

Klapp, S. T., Boches, C. A., Trabert, M. L., \& Logan, G. D. (1991). Automatizing alphabet arithmetic: II. Are there practice effects after automaticity is achieved? Journal of Experimental Psychology: Learning, Memory, \& Cognition, 17, 196-209.

Kliegl, R., Mayr, U., \& Krampe, R. T. (1994). Time-accuracy functions for determining processes and person differences: An application to cognitive aging. Cognitive Psychology, 26, 134-164.

Kyllonen, P. C., \& Christal, R. E. (1990). Reasoning ability is (little more than) working-memory capacity?! Intelligence, 14, 389-433.

Lemaire, P., ABdi, H., \& FAyol, M. (1996). The role of working memory resources in simple cognitive arithmetic. European Journal of Cognitive Psychology, 8, 73-103.

Logan, G. D. (1979). On the use of a concurrent memory load to measure attention and automaticity. Journal of Experimental Psychology: Human Perception \& Performance, 5, 189-207.

LogAN, G. D. (1980). Short-term memory demands of reaction-time tasks that differ in complexity. Journal of Experimental Psychology: Human Perception \& Performance, 6, 375-389.

Logie, R. H., Gilhooly, K. J., \& WynN, V. (1994). Counting on working memory in arithmetic problem solving. Memory \& Cognition, 22, 395-410.

MaYr, U., \& KLIEgL, R. (1993). Sequential and coordinative complexity: Age-based processing limitations in figural transformations. Journal of Experimental Psychology: Learning, Memory, \& Cognition, 19, 1297-1320.

MaYr, U., \& Kliegl, R. (2000). Complex semantic processing in old age: Does it stay or does it go? Psychology \& Aging, 15, 29-43.

Mayr, U., Kliegl, R., \& Krampe, R. T. (1996). Sequential and coordinative processing dynamics in figural transformation across the life span. Cognition, 59, 61-90.

McElree, B. (1998). Attended and non-attended states in working memory: Accessing categorized structures. Journal of Memory \& Language, 38, 225-252.

Salthouse, T. A. (1991). Mediation of adult age differences in cognition by reductions in working memory and speed of processing. Psychological Science, 2, 179-183.

Salthouse, T. A. (1994). The aging of working memory. Neuropsychology, 8, 535-543. 
SAlthouse, T. A. (1996). The processing speed theory of adult age differences in cognition. Psychological Review, 103, 403-428.

SchneIder, W., \& Detweiler, M. (1987). A connectionist/control architecture for working memory. In G. H. Bower (Ed.), The psychology oflearning and motivation (Vol. 21, pp. 53-119). New York: Academic Press.

Shah, P., \& Miyake, A. (1996). The separability of working memory resources for spatial thinking and language processing: An individual differences approach. Journal of Experimental Psychology: General, 125, 4-27.

Verhaeghen, P., Kliegl, R., \& Mayr, U. (1997). Sequential and coordinative complexity in time-accuracy functions for mental arithmetic. Psychology \& Aging, 12, 555-564.

WechSLer, D. (1964). Der Hamburg Wechsler Intelligenztest für Erwachsene (HAWIE). Bern: Huber.

West, R. L. (1996). An application of prefrontal cortex function theory to cognitive aging. Psychological Bulletin, 120, 272-292.

West, R. L. (1999). Age differences in lapses of intention in the Stroop task. Journal of Gerontology: Psychological Sciences, 54B, 3443.

Wickens, C. D. (1991). Processing resources and attention. In D. L.
Damos (Ed.), Multiple-task performance (pp. 3-34). London: Taylor \& Francis.

\section{NOTES}

1. Logical gates combine two binary inputs into one binary output by a logical operation like "and," "or," and others. In the task, several logical gates were combined into small networks that were presented graphically with given inputs, and participants had to compute the outputs.

2. One reviewer noted that Experiment 2 had a smaller sample size than Experiment 1 and therefore had less power to detect an age $\times$ condition interaction. Post hoc power analyses yielded an estimated power for the critical interaction of age with the access contrast of .985 for accuracy and .982 for reaction times in Experiment 1. The power of Experiment 2 to detect interactions with equivalent effect sizes was .973 and .968 for accuracy and reaction times, respectively. The absence of such an interaction in Experiment 2 therefore did not result from a lack of power alone.

(Manuscript received October 27, 1999; revision accepted for publication July 5, 2000.) 